\section{Estado nutricional en niños menores de 5 años del distrito de San Marcos, Ancash, Perú}

\author{
Nutrition status in children \\ aged under 5 in San Marcos \\ district, Ancash, Peru
}

\begin{abstract}
Objective: To assess the nutrition status of a cohort of children aged under five years seen in Carhuayoc and two slums Ayash huaripampa and Pujun in San Marcos district, Ancash, Peru, considered as poor and very poor populations respectively. Subjects and methods: Work sample included 178 children seen between May and December 2011. Variables used were weight $(\mathrm{Kg})$ and height $(\mathrm{cm})$ to measure anthropometric indicators by data Z scores calculated in Anthro program (WHO 2005). The anthropometric measures were made using international standard methodology. Results: Analysis of indicators showed a nourished prevalence in the majority of studied children. Height/age $(H / A)$ $Z<-2$ (moderate) or Heightlage (H/A) $Z<-3$ (severa) parameters was observed $46 \%$ of Carhuayoc children, $60 \%$ of Pujun children and $62 \%$ of Ayash Huaripampa children. Conclusions: These results showed the high rates of malnutrition in the rural population on Ancash, which would be associated with different negative factors in this area. Programs of personal identification of nourished children and nutrition follow up with nutritional foods will need adopted at these places.
\end{abstract}

Key words: Nutrition status, malnutrition, anthropometry.

\section{INTRODUCCIÓN}

La desnutrición infantil es un problema importante a nivel mundial (1). Su etiopatogenia es multifactorial, estando condicionada por el medio socioeconómico en el que crece y se desarrolla el niño (2). La mala nutrición continua siendo un gran problema de salud pública, especialmente en los niños de la primera edad de países subdesarrollados, lo cual contribuye a los elevados índices de morbilidad y mortalidad (3). Muchas son las causas que contribuyen a la existencia de los problemas nutricionales, entre ellos la carencia de nutrientes que no permite cubrir los requerimientos mínimos del organismo y la presencia de infecciones que interfieren en la adecuada utilización de estos $(4,5)$.

Las grandes encuestas nacionales para medir la desnutrición infantil, muestran cifras globales basadas en una población representativa que no permiten apreciar cómo crecen los niños de lugares más pobres del país y que además son beneficiarios de programas de complementación alimentaria.
Víctor Arrunátegui-Correa

Dirección de Investigación, ESKE Corporation SAC,

Lima, Perú

Dirigir la correspondencia a: Profesor

Víctor Arrunátegui-Correa Av. Arequipa 3773-4 San Isidro, Lima Teléfono: 222-4187

Correo electrónico: arruna9@hotmail.com

Este trabajo fue recibido el 21 de Noviembre de 2015 y aceptado para ser publicado el 1 de Abril de 2016.
De esta manera se ha creído conveniente estudiar a todos los niños de hasta 5 años de edad de 3 poblaciones pobres del CP Carhuayoc incluyendo Carhuayoc que participan en este programa.

Los niños que participaron en el estudio fueron parte de un proyecto de prácticas de alimentación saludable aplicada en el Centro Poblado (CP) de Carhuayoc que es el más populoso CP del Distrito de San Marcos y está ubicado en la sierra norte en el flanco oriental de la cordillera blanca. Se encuentra a una altura media de 3,150 msnm. Según datos estadísticos, su población asciende a 2000 habitantes donde el 50\% de sus habitantes son niños y adolescentes de 0 a 20 años.

El objetivo del presente estudio fue analizar comparativamente, los resultados de las evaluaciones antropométricas de los niños menores de 5 años de cada una de las 3 poblaciones del CP Carhuayoc. Esto servirá como estudio que permita identificar el riesgo nutricional que afecta a muchos niños realizando acciones de detección-acción y planificar políticas 
públicas orientadas a corregir los problemas relacionados con la alimentación y nutrición de cada niño en particular.

\section{SUJETOS Y MÉTODOS}

Descripción del área de estudio

EI CP Carhuayoc en el Distrito de San Marcos se encuentra ubicado en la sierra de Ancash del Perú, a una altura de 3100 msnm. Este CP cuenta con 7 caseríos, Pujun, Ayash Huaripampa, Pacash, Manyanpampa, Ango, Juprog y Chuchupampa. Cuenta con un total de 2000 habitantes y con una población de más del $50 \%$ entre niños y adolescentes de hasta 19 años.

\section{Diseño del estudio}

Se realizó un estudio descriptivo de corte transversal. El universo de trabajo estuvo conformado por 178 niños menores de 5 años en el periodo comprendido entre mayo y diciembre 2011.

Para evaluar el estado nutricional mediante indicadores antropométricos se utilizó la metodología internacionalmente aceptada incluida en la guía elaborada por EL Centro Nacional de Alimentación y Nutrición (CENAN) (6), utilizándose balanzas digitales calibradas con precisión de $100 \mathrm{~g}$ y tallimetros con precisión de $0.1 \mathrm{~cm}$ modelo OMS confeccionados de acuerdo con las especificaciones técnicas de este mismo centro (7).

Los datos se registraron en una planilla especialmente confeccionada para el estudio con fecha de intervención, sexo, fecha de nacimiento, talla y peso. La edad se calculó en base a la fecha de nacimiento y la fecha de consulta. De acuerdo a la OMS, la medición de los niños mayores de 2 años fue realizada en pie.

Para la expresión e interpretación de los datos antropométricos se utilizó el cómputo de puntuaciones $Z$. Los índices $Z$ seleccionados, del peso para la talla $(\mathrm{P} / \mathrm{T})$, peso para la edad $(P / E)$ y talla para la edad ( $T / E)$ se calcularon mediante el paquete estadístico WHO ANTHRO 2005. Se realizó el análisis conjunto de los índices Z P/T, Z P/E y Z T/E empleando la tabla de clasificación propuesta por la OMS. Se consideró evidencia de desnutrición a la presencia de uno o más índices antropométricos por debajo de $-2 Z$ (8).

Un puntaje $Z<-3$ generalmente está asociado a trastornos severos de nutrición (desnutrición severa o grave) y un puntaje $Z<-2$ identifica a los desnutridos moderados que en esta publicación son considerados también desnutridos.

\section{Consideraciones éticas}

El consentimiento informado fue obtenido oralmente de los padres a quienes se les informó sobre la naturaleza del trabajo y los beneficios del mismo. La Gerencia de Desarrollo Humano del distrito de San Marcos, participó en la concientización de la población con avisos difundidos días previos al inicio de este estudio.

\section{RESULTADOS}

El total de los niños estudiados en el CP Carhuayoc (Carhuayoc, Pujun y Ayash Huaripampa) fueron 178 niños. De los 178 niños pesados y tallados, 83 (46.63\%) fueron de Carhuayoc, 50 (28.09\%) del caserío Pujun y 45 (25.28\%) del caserío Ayash Huaripampa.

La tabla 1 muestra que en Carhuayoc ( $n: 83$ ), la edad de los niños vario con predominio del grupo de nacidos el año 2008 (hasta 3 años de edad). Esta tabla muestra también que la distribución por grupo de edad fue: 6 (7.23\%) de hasta 1 año de edad, 16 (19.27\%) de hasta 2 años de edad, 28 (33.13\%) de hasta 3 años de edad, 21 (25.3\%) de hasta 4 años de edad y $12(14.46 \%)$ de hasta 5 años de edad.

La desnutrición grave afectó a 10 (12.1\%) niños, la desnutrición moderada a 28 (33.7\%) y el sobrepeso/obesidad se presentó en sólo 4 (4.8\%) de los niños estudiados en Carhuayoc. Se consideraron eutróficos a 41 (49.4\%) niños. Los dos tipos de desnutrición grave o moderada fue más frecuente en los niños nacidos el año 2008: 3 años de edad (tabla 1).

De los 10 niños con desnutrición grave con estaturas tan bajas como Z T/E Z <-3 o Z T/E Z <-4, 3 (30\%) fueron del sexo femenino y 7 (70\%) del sexo masculino. De los 28 niños con desnutrición moderada: Z T/E Z <-2, 14 (50\%) fueron del sexo femenino y 14 (50\%) del sexo masculino.

La tabla 2 muestra que en el caserío Pujun (n: 50), la edad de los niños varió con ligero predominio del grupo de nacidos el año 2007 (hasta 4 años de edad). La tabla muestra también que la distribución por grupo de edad fue: 10 (20.0\%) de hasta 1 año de edad, 10 (20.0\%) de hasta 2 años de edad, 12 (24.0\%) de hasta 3 años de edad, 15 (30.0\%) de hasta 4 años de edad y 03 (6.0\%) de hasta 5 años de edad.

La desnutrición grave afecto a 12 (24.0\%) niños, la desnutrición moderada a $18(36.0 \%)$ y el sobrepeso/obesidad se presentó en sólo 3 (6.0\%) niños estudiados en el caserío Pujun. Se consideraron eutróficos a 17 (34.0\%) niños. Los dos tipos de desnutrición grave o moderada fueron más frecuentes en los niños nacidos los años 2007 y 2008: 4 y 3 años de edad respectivamente (tabla 2 ).

De los 12 niños con desnutrición grave, 9 (75.0\%) fueron del sexo femenino y 3 (25.0\%) del sexo masculino. De los 18 niños con desnutrición moderada, 10 (56.0\%) fueron del sexo femenino y 8 (44.0\%) del sexo masculino.

TABLA 1

Estado nutricional de niños menores de 5 años de Carhuayoc, según edad.

\begin{tabular}{|c|c|c|c|c|}
\hline AÑO DE & EUTROFICOS & DESNUTRICIÓN & DESNUTRICIÓN & SOBREPESO \\
\hline NACIMIENTO & & GRAVE & MODERADA & OBESIDAD \\
\hline 2006 & $6(14.6 \%)$ & $1(10.0 \%)$ & $5(17.8 \%)$ & $0(0.0 \%)$ \\
\hline 2007 & $11(26.8 \%)$ & $3(30.0 \%)$ & $7(25.0 \%)$ & $0(0.0 \%)$ \\
\hline 2008 & $10(24.4 \%)$ & $4(40.0 \%)$ & $13(46.4 \%)$ & $1(25.0 \%)$ \\
\hline 2009 & $9(22.0 \%)$ & $2(20.0 \%)$ & $3(10.7 \%)$ & $2(50.0 \%)$ \\
\hline 2010 & $5(12.2 \%)$ & $0(0.0 \%)$ & $0(0.0 \%)$ & $1(25.0 \%)$ \\
\hline TOTAL & 41 & 10 & 28 & 04 \\
\hline
\end{tabular}


La tabla 3 muestra que en el caserío Ayash Huaripampa (n: 45), la edad de los niños varió con predominio del grupo de nacidos el año 2009 (hasta 2 años de edad). La tabla muestra también que la distribución por grupo de edad fue: 12 (7.23\%) hasta 1 año de edad, 16 (16.87\%) hasta 2 años de edad, 06 (36.15\%) hasta 3 años de edad, 08 (25.3\%) hasta 4 años de edad y $03(14.46 \%)$ hasta 5 años de edad.

La desnutrición grave afectó a 9 (20.0\%) niños, la desnutrición moderada a 19 (43.0\%) y el sobrepeso/obesidad se presentó en sólo 1 (1.0\%) niño estudiado en el caserío Ayash Huaripampa. Se consideraron eutróficos a 16 (36.0\%) niños. Los dos tipos de desnutrición grave o moderada fue más frecuentes en los niños nacidos el año 2009: 2 años de edad (44\%) y los nacidos en 2008: 3 años de edad (33\%), mientras que la desnutrición moderada fue más frecuente en los nacidos el año 2009: 2 años de edad (37\%) y los nacidos en el año2007: 4 años de edad (32\%) (tabla 3).

De los 9 niños con desnutrición grave, 5 (55.6\%) fueron del sexo femenino y 4 (44.4\%) del sexo masculino. De los 19 niños con desnutrición moderada, 5 (26.3\%) fueron del sexo femenino y 14 (73.7\%) del sexo masculino.

\section{DISCUSIÓN}

El estado nutricional de una persona es la resultante entre la ingesta y los requerimientos de nutrientes. En los niños y durante los 3 primeros años de vida, debido a la .gran velocidad de crecimiento, cualquier factor que altere este equilibrio, repercute rápidamente en él. Por esta razón el control periódico de ellos constituye el elemento más valioso para la detección precoz de alteraciones nutricionales y permite obtener una evaluación oportuna y adecuada. La forma más fácil, económica y universalmente aplicable para observar el crecimiento físico es la antropometría (9).

Como se puede apreciar, Ayash Huaripampa presento el grado más alto de desnutrición infantil razón por la cual los niños de solo 2 años de edad muestran parámetros de desnutrición. En el Perú, entre 1991 y el 2005 la tasa de retardo de crecimiento infantil se ha mantenido alta y no ha variado desde 1996 (10). El Perú es un país con una de las más altas tasas de prevalencia de retardo de crecimiento infantil después de Guatemala, con tasas similares a Bolivia y Haití (11). Esto puede deberse a que las estrategias aplicadas para luchar contra la desnutrición no son las más indicadas.

Este estudio hizo parte de un proyecto de inversión pública con Código SNIP 92713 de la Municipalidad de San Marcos "FORTALECIMIENTO DE CAPACIDADES EN PRÁCTICAS SALUDABLES RELACIONADAS CON LA ALIMENTACIÓN Y NUTRICIÓN EN EL CENTRO POBLADO DE CARHUAYOC, DISTRITO DE SAN MARCOS - HUARI - ANCASH" que duro 3 años (2009-2011) y ayudo a identificar el alto grado de retardo en el crecimiento existente en el CP Carhuayoc.

Los resultados antropométricos reflejan que en Carhuayoc, la forma predominante de desnutrición, al igual que en otras partes del mundo subdesarrollado es el factor Z T/E menor de -2 (12). El caso particular de retardo en el crecimiento Z T/E encontrado en $46.0 \%$ de los niños en Carhuayoc, 60.0\% en Pujun y $62.0 \%$ en Ayash Huaripampa, se relaciona con antecedentes de mala alimentación, malas condiciones higiénicas y la presencia de enfermedades que conllevan al deterioro del estado nutricional $(13,14)$. El estudio de Guevara Linares en el distrito de Chavin de Huantar (15) que es cercano a Carhuayoc muestra también el alto grado de desnutrición

TABLA 2

Estado nutricional de niños menores de 5 años de Pujun, según grupos de edad.

\begin{tabular}{|c|c|c|c|c|}
\hline AÑO DE & EUTROFICOS & DESNUTRICIÓN & DESNUTRICIÓN & SOBREPESO \\
\hline NACIMIENTO & & GRAVE & MODERADA & OBESIDAD \\
\hline 2006 & $0(0.0 \%)$ & $2(16.7 \%)$ & $0(0.0 \%)$ & $1(33.3 \%)$ \\
\hline 2007 & $6(35.2 \%)$ & $3(25.0 \%)$ & $6(33.4 \%)$ & $0(0.0 \%)$ \\
\hline 2008 & $3(17.7 \%)$ & $3(25.0 \%)$ & $6(33.4 \%)$ & $0(0.0 \%)$ \\
\hline 2009 & $5(29.4 \%)$ & $2(16.7 \%)$ & $2(11.1 \%)$ & $1(33.3 \%)$ \\
\hline 2010 & $3(17.7 \%)$ & $2(16.7 \%)$ & $4(22.2 \%)$ & $1(33.3 \%)$ \\
\hline TOTAL & 17 & 12 & 18 & 03 \\
\hline
\end{tabular}

TABLA 3

Estado nutricional de niños menores de 5 años de Ayash Huaripampa, según grupos de edad.

\begin{tabular}{|c|c|c|c|c|}
\hline AÑO DE & EUTROFICOS & DESNUTRICIÓN & DESNUTRICIÓN & SOBREPESO \\
\hline NACIMIENTO & & GRAVE & MODERADA & OBESIDAD \\
\hline 2006 & $1(6.2 \%)$ & $2(22.2 \%)$ & $0(0.0 \%)$ & $0(0.0 \%)$ \\
\hline 2007 & $2(12.5 \%)$ & $0(0.0 \%)$ & $6(31.6 \%)$ & $0(0.0 \%)$ \\
\hline 2008 & $1(6.2 \%)$ & $3(33.3 \%)$ & $2(10.5 \%)$ & $0(0.0 \%)$ \\
\hline 2009 & $5(31.3 \%)$ & $4(44.4 \%)$ & $7(36.8 \%)$ & $0(0.0 \%)$ \\
\hline 2010 & $7(43.8 \%)$ & $0(0.0 \%)$ & $4(21.1 \%)$ & $1(100.0 \%)$ \\
\hline TOTAL & 16 & 09 & 19 & 01 \\
\hline
\end{tabular}


existente en la zona (63\%).

El proceso que conduce a una T/E baja puede iniciarse en la vida intrauterina lo que implicaría en el buen cuidado que debe tener la madre gestante en cuanto a nutrición. Una estrategia promisoria para prevenir estos defectos debería ser el suplemento alimenticio con múltiple micronutrientes (MMN) más que fierro y ácido fólico (16). Estos estudios confirman que la T/E es el índice antropométrico que mejor refleja las condiciones sociales globales de los caseríos de Carhuayoc, ya que el retardo en el crecimiento infantil es mayor en los estratos más pobres y rurales.

La información obtenida permite visualizar esquemas de seguimiento. Las altas cifras de desnutrición en las comunidades rurales estudiadas nos permiten afirmar que la prevención mediante antropometría entre 2 o más medidas distanciadas en el tiempo (lo ideal es 6 meses) junto con una atención especializada y alimentación complementaria saludable, constituyen medidas básicas para el control en futuras intervenciones en poblaciones rurales. Los niños diagnosticados con desnutrición moderada son los niños en riesgo, así: 34.0\% en Carhuayoc, $36.0 \%$ en Pujun y $42 \%$ en Ayash Huaripampa, son los llamados a hacerles un seguimiento con suplemento alimenticio especifico además de complementar con actividades educativas para las madres y familias conjuntamente con infraestructura sanitaria básica que disminuirá las altas tasas de parasitismo en los niños encontrados en este mismo distrito (17) similares a otros estudios en áreas urbanas pobres de la capital Lima (18).

\section{RESUMEN}

Objetivo: Evaluar el estado nutricional de niños menores de 5 años que viven en el centro poblado de Carhuayoc y dos de sus caseríos: Pujun y Ayash Huaripampa, en el Distrito de San Marcos, Departamento de Ancash, Perú, considerados de pobreza y pobreza extrema, respectivamente. Sujetos y métodos: El universo de trabajo estuvo conformado por 178 niños atendidos entre mayo y diciembre del 2011. Se emplearon las variables: peso $(\mathrm{Kg})$ y talla $(\mathrm{cm})$ para medir indicadores antropométricos para lo cual se utilizó el cómputo de puntuaciones Z mediante el programa Anthro (OMS 2005). Las mediciones antropométricas se realizaron de acuerdo con la metodología estándar internacional. RESULTADOS: Los indicadores mostraron una prevalencia alta de desnutrición moderada o grave en la mayoría de los niños estudiados. El parámetro Talla/edad (T/E) $Z<-2$ (moderada) o (T/E) Z<-3 (grave) se observó en $46 \%$ de los niños de Carhuayoc, 60\% de los niños de Pujun y $62 \%$ de los niños de Ayash Huaripampa. CONCLUSIONES: Estos resultados demuestran el alto índice de desnutrición en la población rural de la sierra de Ancash, debido a diferentes factores negativos en esta zona. Una identificación personal de desnutridos graves y seguimiento suplementario con alimentos nutritivos, debería ser necesario.

Palabras claves: Estado nutricional, desnutrición, antropometría.

Agradecimientos: A la obstetra Erika Pantoja Domínguez, a los enfermeros Cristina Loarte Guerra, y Larry Castillo Pérez y a la técnica en enfermería Cecilia Zorrilla Bañez, por su apoyo en el pesaje y medición de los niños y en las charlas sobre prácticas saludables a los padres de familia.

\section{BIBLIOGRAFÍA}

1. Waterloo J, Tomkins A, Granthan-McGregor M. Malnutrition and mortality. In: Malnutrition and mortality. OPS: Scientific Publication No. 555; 1996. Pp.401-422.

2. De Onis M, Monteiro C, Akre J. The worldwide magnitude of protein-energy malnutrition: an overview fron the WHO global database on child growth, Bull World health Organ. 1993; 71: 703-12.

3. Castro J, García E, Castro E, Mejía E. Nutritional evaluation and prevalence of parasitism in poverty areas, I: altitude, Rev Peruana Med Trop U.N.M.S.M. 1991; 5:67-74.

4. Ferreira $M$, Souza $W$, Perez E, Lapa T, Carvalho A, Furtado $A$, Coutinho $H$, Wakelin D. Intestinal Helminthiasis and Anemia in Youngsters from Matriz da Luz, District of Sao Lourenco da Mata, State of Pernambuco, Brazil, Mem Inst Oswaldo Cruz 1998; 93(3): 289-93.

5. Solano L, Acuna I, Baron M, Moron de Salim A, Sanchez A. intestinal parasitic infestation and others infectious backgrounds influence in the antropometric nutritional status of children in poverty, Parasitol Latinoam 2008; 63: 12-9.

6. National Institute of Health, National Center for Food and Nutrition. The measurement of height and weight. Guidance for health primary care. Lima: INS; 2004.

7. WHO. Child Growth Standards WHO. Geneva: Departamento de nutrición para la salud y el desarrollo-OMS; 2006. Disponible en http:/www.who.int/childgrowth/es/

8. OMS. Physical status: the use and interpretation of anthropometry. Washington: OPS, OMS, 1995 (Technical Report Series; 854).

9. Rojas M. practical aspects of pediatric anthropometry, Paediatrica 2000; 3(1): 22-6.

10. Mispireta $M$, Rosas A, Velasquez J, Lescano A, Lanata C. nutritional transition in Peru, 1991-2005, Rev Peru Med Exp Salud Pública 2007; 24(2): 129-35.

11. Barria $P$, Mauricio $R$, Amigo $H$. nutrition transition: a review of Latin American profile, ALAN 2006; 56(1): 3-11.

12. De Onis $M$, Frongillo $E$, Blossner $M$. Is malnutrition declining? An analysis of changes in levels of child malnutrition since 1980, Bull WHO 2000; 78:1222- 33.

13. Blumenthal D, Schultz M. Effects of Ascaris Infection on Nutritional Status in Children, Am J Trop Med Hyg. 1976; 25(5): 682-90.

14. Oberhelman R, Guerrero E, Fernández A. Correlation between intestinal parasitosis, physical growth and psychomotor development among infants and children from rural Nicaragua, Am J Trop Med Hyg. 1998; 58: 470-475.

15. Guevara Linares $X$. nutritional status of children under 5 years of rural communities and urban neighborhoods in the district of Chavin- 2003, Pediatrica 2003; 5(1): 14-20.

16. Zerfu T, Ayele $H$, Micronutrientes and pregnancy: Effect of supplementation on pregnancy and pregnancy outcomes: a systematic reviews Nutr. 2013; 12:20.

17. Jacinto $E$, Aponte $E$, Arrunátegui-Correa V. Prevalence of intestinal parasites in children of different levels of education in the district of San Marcos, Ancash, Peru. Rev Med Herediana 2012; 23(4):235-9.

18. Ubillus G, Ascarrus A, Soto C, Medina J, Falconi R. et al. Enteroparasitosis and nutritional status in children under 4 years of Wawa Wasi of Pamplona Alta San Juan de Miraflores Lima Peru. Rev. Horizonte Med. 2008; 8(2):36-40. 